sensory deprivation typically affecting the anterior visual pathway (due to cataract or senile macular degeneration) and advanced age (older than 60 years). As the hallucinatory symptoms in CBS occur with preserved insight, these are not true but pseudohallucinations.

The syndrome occurs as a result of the dissociation between visual perception and visual sensory input in psychologically normal aged people. Functional neuroimaging (fMRI) has shown that the hallucinations of colour, faces, textures, and objects in CBS correlate with the cerebral activity in the ventral extrastriate visual cortex whereas the contents of hallucinations reflect the functional specialisation of this region. ${ }^{2}$ In our experience, carbamazepine has been partially effective in suppressing the visual pseudohallucinations of CBS, presumably because it might inhibit the increased ventral extrastriate neuronal activity in patients with CBS that persists between the attacks of hallucinatory symptoms. ${ }^{2}$

In Cole's case, ${ }^{1}$ the occipital infarct in the dominant (left) hemisphere led to the dissociation between the visual sensory input (now limited to the right striate cortex) and the visual perception sense of the dominant extrastriate and the visual association cortex spared by the ischaemic event. Collicular vision ("blindsight") plays no part in the symptoms of visual hallucination. In the light of the recent fMRI data, CBS may be considered as a visual dissociation syndrome similar to the cortical dissociation syndromes well recognised in the Geschwind model of language function. Cole gives one of the finest clinical examples to illustrate this phenomenon. Why CBS should occur with visual sensory deprivation exclusively in elderly people and not in young people is unknown but it might reflect the nature of neural plasticity in the visual cortex as opposed to the other cortical functions.

We were, however, a little surprised that CBS did not feature even once in the otherwise erudite discussion of the case report. ${ }^{1}$ Did Cole and the reviewers of the fournal not consider this common diagnostic possibility?

ABHIJIT CHAUDHURI

Department of Neurology, University of Glasgow, UK

1 Cole $M$. When the left brain is not right the right brain may be left: report of personal experience of occipital hemianopia. $\mathcal{F}$ Neurol Neurosurg Psychiatry 1999;67:169-73.

2 Ffytche DH, Howard RJ, Brammer MJ, et al. The anatomy of conscious vision: an fMRI study of visual hallucinations. Nat Neurosci 1998;1:738-42.

\section{Corticobasal ganglionic degeneration and/or frontotemporal dementia?}

I read with interest the recent paper by Mathuranath et $a l^{1}$ describing two patients with the pathology of corticobasal ganglionic degeneration (CBD), the first presenting with the syndrome of frontotemporal dementia and the second with a mixed picture dominated by progressive aphasia. The concept that CBD may present with clinical features distinct from the "perceptuomotor" syndrome widely recognised as "classic" of the disorder is not new. However, until recently it had generally been thought that these cases represented the minority and thus could be considered "atypical". However, knowledge about this disorder has evolved since our group and others reported the first large series. I think that in 2000 it is not appropriate to quote our 1994 book chapter ${ }^{2}$ stating that "Frank dementia or language dysfunctions are said to be rare and, if present, are mild, and typically occur late in the course of the disease," 1 as current wisdom. Within 2 years of that book chapter ourselves and others were reporting alternative presentations for this pathology and most recently we have published the clinicalpathological experience of the Canadian Brain Tissue Bank in a paper that was presumably in press at the time that the paper of Mathuranath et al was being reviewed. ${ }^{3}$ In this study we found that of 13 patients proved pathologically to have CBD, nine presented with cognitive or language disturbances, only one failed to show dementia during the course of the illness and only four were diagnosed as having CBD in life. Since that time, a 14 th patient has come to necropsy whose presentation was that of primary progressive aphasia.

There have also been patients reported in the literature by ourselves and others with alternative neuropathological disorders presenting with clinical features that were mistaken for the "classic" (but now it seems not the commonest) phenotype of CBD including progressive supranuclear palsy, Pick's disease, motor neuron inclusion body dementia, Alzheimer's disease, and familial frontotemporal dementia due to chromosome 17 mutations.

In summary, clinical and pathological experience at the turn of the century strongly supports the conclusions of Mathuranath et al. Clinical phenotypes have not proved to be restricted to specific pathological substraits and several different clinical phenotypes may be caused by the same underlying pathology, probably largely dependent on the anatomical distribution of greatest involvement. Where I mainly take issue with the authors is in their belief, which largely justifies their report, that a clear distinction between CBD and FTD is "currently accepted". The cumulative literature since our 1994 review, most recently culminating in a monograph on a topic, ${ }^{4}$ indicates that CBD is no longer thought of as a predominately extrapyramidal disorder that is distinct and unrelated to frontotemporal dementia.

ANTHONY E LANG
Toronto Western Hospital, Toronto, Canada

1 Mathuranath PS, Xuereb JH, Bak T, et al. Corticobasal ganglionic degeneration and/or frontotemporal dementia? A report of two overlap cases and review of literature. $f$ Neurol Neurosurg Psychiatry 2000;68:304-12.

2 Lang AE, Riley DE, Bergeron C. Cortical-basa ganglionic degeneration. In: Calne $\mathrm{DB}$, ed Neurodegenerative diseases. Philadelphia: WB Saunders, 1994:877-94.

3 Grimes DA, Lang AE, Bergeron CB. Dementia as the most common presentation of corticalbasal ganglionic degeneration. Neurology 1999 53:1969-74.

4 Litran I, Goetz CG, Lang AE. Corticobasal degeneration and related disorders. Philadelphia, degeneration and related disorders. Philadelphia,
PA: Lippincott Williams and Wilkins, 2000.

\section{Diagnostic criteria for corticobasal degeneration}

The study of Mathuranath et al $l^{1}$ of corticobasal degeneration (CBD) and its overlap with frontotemporal dementia (FTD) contributes interesting information to a controversial area of neurodegeneration. Unfortunately it also introduces a potentially confusing histopathological diagnostic error. The authors found oligodendroglial inclusions which they considered to be glial cytoplasmic inclusions and claimed that these, diagnostic hallmark of multiple system atrophy, "have been described in other neurodegenerative diseases, including CBD". This is clearly not the case.

To support their view the authors refer to two papers $^{23}$ reporting, among other cytoskeletal abnormalities, tau positive inclusions in oligodendroglial cells. A letter, published earlier in this fournal, ${ }^{4}$ but not quoted in the current paper, ${ }^{1}$ has also claimed that glial cytoplasmic inclusions are not exclusive to multiple system atrophy. The evidence is now overwhelming that they are. What the authors of this ${ }^{1}$ and the other papers $^{2-4}$ have described are indeed tau positive oligodendroglial inclusions, but they are not the same as glial cytoplasmic inclusions. ${ }^{5}$ Oligodendroglial inclusions, chiefly coiled bodies, undoubtedly occur in various neurodegenerative diseases, including CBD, but their morphology and molecular pathology are different from those of glial cytoplasmic inclusions. Whereas glial cytoplasmic inclusions immunostain with $\alpha$-synuclein ${ }^{6}$ and only with unphosphorylated tau antibodies, ${ }^{7}$ the oligodendroglial inclusions seen in CBD and other neurodegenerative diseases are $\alpha$-synuclein negative and give positive reaction with both phosphorylated and unphosphorylated tau antibodies. This basic difference has been recognised by a new classification of neurodegenerative disorders: $\mathrm{CBD}$ is one of the tauopathies, whereas multiple system atrophy is an $\alpha$-synucleinopathy. Thus glial cytoplasmic inclusions remain the most consistent and reliable diagnostic hallmarks of multiple system atrophy and do not occur in other neurodegenerative diseases.

P L LANTOS

Institute of Psychiatry, King's College London, Department of Neuropathology, London SE5 8AF, UK

1 Mathuranath PS, Xuereb JH, Bak T, et al. Corticobasal ganglionic degeneration and/or frontotemporal dementia? A report of two overlap cases and review of literature. $\mathcal{F}$ Neurol Neurosurg Psychiatry 2000;68:304-12.

2 Wakabayashi K, Oyanagi K, Makifuchi $\mathrm{T}$, et al . Corticobasal degeneration: etiopathological significance of the cytoskeletal alterations. Acta Neuropathol 1994;87:545-53.

3 Mori H, Nishimura M, Namba Y, et al. Corticobasal degeneration: a disease with widespread appearance of abnormal tau and neurofibrillary tangles, and its relation to progressive supranuclear palsy. Acta Neuropathol 1994;88: 113-21.

4 Daniel SE, Geddes JF, Revesz T. Glial cytoplasmic inclusions are not exclusive to multiple system atrophy. $\mathcal{F}$ Neurol Neurosurg Psychiatry 1995;58:262

5 Papp MI, Kahn JE, Lantos PL. Glial cytoplasmic inclusions in the CNS of patients with multiple system atrophy (striatonigral degeneration, olivopontocerebellar atrophy and ShyDrager syndrome). $\mathcal{F}$ Neurol Sci 1989;94:79100.

6 Spillantini MG, Crowther RA, Jakes R, et al. Filamentous $\alpha$-synuclein inclusions link multiple system atrophy with Parkinson's disease and dementia with Lewy bodies. Neurosci Lett 1998;251:205-8.

7 Cairns NJ, Mackay D, Daniel SE, et al. Tau-pathology of oligodendroglial inclusions in multiple system atrophy, progressive supranuclear palsy and corticobasal degeneration. Neuropathol App Neurobiol 1998;24:145.

Xuereb and Hodges reply

The topic of how to apply the term "glial cytoplasmic inclusions" and their specificity to a particular disorder is clearly controversial and in a state of evolution. We used glial cytoplasmic inclusions in a non-specific way to indicate simply the presence of cytoplasmic inclusions in glial cells. We found these 
inclusions in subcortical oligodendrocytes. Glial inclusions were initially described in multiple system atrophy by Papp et al in $1989 ;{ }^{1}$ their paper brought glial cellular pathology in neurodegenerative disease to the attention of neuropathologists. Cytoplasmic inclusions in glial cells have since been reported in various neurodegenerative diseases. The label "glial cytoplasmic inclusions" and the initials "GCIs" used in the general sense cannot, therefore, properly be regarded as pathognomonic of any single disease entity. Indeed, a neuroscientist without neuropathological training could conceivably misdiagnose tissue as coming from a case of multiple system atrophy if that tissue contained oligodendroglial cytoplasmic inclusions (in silver preparations or ubiquitin immunohistochemistry). Our paper does not, we would argue, contain a "histopathological diagnostic error" as suggested by Lantos.

On the other hand, Lantos' criticism that we made no mention of recent discoveries of $\alpha$-synuclein involvement in the biology of MSA is justified. In the discussion, we should have emphasised the fact that glial cytoplasmic inclusions in multiple systems atrophy, and so far only in multiple systems atrophy, are indeed $\alpha$-synuclein-positive and phosphorylated tau negative, whereas the opposite is true for the glial cytoplasmic inclusions of $\mathrm{CBD}$ and related tauopathies. Lest the future should see $\alpha$-synuclein positive glial cytoplasmic inclusions identified in some other disease, it is well to emphasise that the diagnosis of multiple systems atrophy depends on the clinical history and distribution pattern of neurodegeneration (which determines the clinical phenotype), and the presence of $\alpha$-synuclein positive glial cytoplasmic inclusions is valuable confirmatory evidence in this context.

We also appreciate Lang's comments on the paper and are very pleased that the cumulative Canadian experience mirrors the conclusions of our paper in that language and/or other cognitive disturbances are a virtually universal feature of CBD and in many cases may indeed be the mode of presentation as highlighted by the recent paper by Grimes et al which appeared after the submission of our manuscript.

JOHN XUEREB JOHN HODGES

MRC Cognition and Brain Sciences Unit, 15 Chaucer Road, Cambridge CB2 2EF, UK

Correspondence to: Professor John Hodges

1 Papp MI, Kahn JE, Lantos PL. Glial cytoplasmic inclusions in the CNS of patients with multiple system atrophy (striatonigral degeneration, olivoponto-cerebellar atrophy, and eration, olivoponto-cerebellar atrophy, and
Shy-Dragar syndrome). $\mathcal{f}$ Neurol Sci 1989: 94:79-100

2 Grimes DA, Lang AE, Bergeron CB. Dementia as the most common presentation of corticalbasal ganglionic degeneration. Neurology 1999, 53:1969-74.

\section{Assessment and treatment of dizziness}

In a recent editorial, Halmagyi and Cremer consider Menière's disease in their discussion of recurrent spontaneous vertigo. ${ }^{1}$ Menière's disease is a diagnosis of exclusion. Many conditions may present with the triad of hearing loss, vertigo, and tinnitus, most importantly vestibular schwannomas. ${ }^{2}$ Gadolinium enhanced MRI imaging is the current gold standard for diagnosing vestibular schwannoma and is mandatory before giving somebody the diagnosis of Menière's disease. The
Committee on Hearing and Equilibrium of the American Academy of OtolaryngologyHead and Neck Surgery have set out guidelines for the diagnosis and evaluation of therapy in Menière's disease. ${ }^{3}$

Audiovestibular testing can be useful in Menière's disease. Caloric testing has poor sensitivity and specificity in diagnosing the disease. Electrocochleography and glycerol dehydration testing can be useful in the earlier stages of the disease before the hearing function is irreversibly and severely lost. In Menière's disease, the most common findings on electrocochleography are an increased summating potential to action potential ratio, a widened summating potential/action potential complex, and a disturbed cochlear microphonic potential. ${ }^{4}$

For medical treatment, dietary advice with strict sodium restriction is useful. However, betahistine probably helps more patients with Menière's disease than any other drugs. Labyrinthine sedatives are also helpful in patients who have severe attacks of vertigo. ${ }^{5}$ Surgical treatment of Menière's disease treats only the vertigo. A wide range of operations have been described, from grommet insertion to vestibular nerve section, all of which have had a similar degree of success. These are particularly difficult to compare due to the huge variations in the natural history of the disease.

Assessment and treatment of patients with balance disturbance covers many specialties, who all approach the problem from slightly different angles with different perspectives. It is important to liase closely with colleagues in associated specialties to optimise the diagnosis and treatment of these patients.

A P COATESWORTH

Department of Otolaryngology, Leeds General Infirmary, Great George Street, Leeds LS1 3EX, UK

1 Halmagyi GM, Cremer PD. Assessment and treatment of dizziness. I Neurol Neurosurg treatment of dizziness.
Psychiatry 2000;68:129-36.

2 Ramsden RT. Vestibular schwannoma. In: ScottBrown's otolaryngology 6th ed. Oxford: Brown's otolaryngology 6 th $\mathrm{ed}$. Oxf O Oxt
Butterworth-Heinemann 1997:3/21/1-38.

3 Committee on Hearing and Equilibrium. Guidelines for the diagnosis and evaluation of therapy in Menière's disease. Otolaryngol Head Neck Surg 1995;113:181-5.

4 Gibson WPR, Moffat DA, Ramsden RT. Clinical electrocochleography in the diagnosis and management of Meniere's disorder. Audiology 1977;16:389-401.

5 Brookes GB. The pharmacological treatment of Menière's disease. Clin Otolaryngol 1996;21:311.

Halmagyi and Cremer reply:

One cannot help but feel a certain sense of nostalgia reading Coatesworth's textbook description of Menière's disease: if only the real world was like that. We deal with his comments in order.

Menière's disease is a diagnosis of exclusion - It is difficult to conceive what needs to be excluded in a patient who has repeated devastating attacks of acute spontaneous vertigo lasting several hours as well as unilateral tinnitus, aural fullness, and fluctuating hearing loss. When the audiogram shows a unilateral low frequency sensorineural hearing loss, the caloric test shows a canal paresis and the electrocochleogram shows a pathologically large summating potential to action potential ratio the patient has Menière's disease. Menière's disease is a clinical diagnosis supported by laboratory testing. It is no more a diagnosis of exclusion than is multiple sclerosis.

The vestibular schwannoma story-This is a difficult one. Maybe the answer is that if someone else is paying and the lawyers are watching anyone with any unilateral balance or hearing problem should have a gadolinium enhanced MRI in case they are harbouring what might eventually become a symptomatic intracanalicular vestibular schwannoma ("acoustic neuroma"). If the patient actually does have one, then the problem becomes not so much the dizziness but what to do about the "tumour". Vestibular schwannomas can, very rarely, present with one, at the most two attacks of acute spontaneous vertigo, ${ }^{2}$ just as they can, rarely, present as sudden hearing loss. ${ }^{2}$ We see about 2500 new patients each year in our balance disorders clinic and in the past 15 years we have seen three patients with acute spontaneous vertigo who had small vestibular schwannomas.

Caloric testing has poor sensitivity and specifcity in diagnosing Menière's disease - In part it depends who does it. Technical standards for caloric testing are in general not as rigorously enforced as those for audiological testing. Some factors that can have a profound influence on the quality of the results include: (a) method of recording-DC electrooculography versus infrared and video methods; (b) method of removing fixation-eye closure versus darkness; (c) method or irrigation-water versus air. ${ }^{3}$ Caloric testing (or "ENG") is no more specific for any disease than is EMG. It shows the site of lesion not the nature of the lesion. When one is confident in the technical standards of the caloric testing the most helpful finding in the diagnosis of Menière's disease is a fluctuating unilateral vestibular loss - the vestibular equivalent of the classic fluctuating hearing loss.

Electrocochleography (ECOG)-Again, it depends on who does it. An ECOG with extratympanic recording of the responses to clicks alone is generally useless or worse, misleading. A transtympanic recording of the responses to tone bursts can disclose, unequivocally, the presence of endolymphatic hydrops, the pathophysiological basis of Menière's disease. ${ }^{4}$ The test is, as Coatesworth notes, most useful in the early stages before a severe fixed hearing loss due to loss of hair cells.

Surgery for Menière's disease-Vestibular nerve section stops the vertigo attacks without worsening the hearing but might cause chronic vestibular insufficiency. ${ }^{5}$ Intratympanic gentamicin is a lot simpler and safer and might be just as good at stopping the vertigo but can worsen the hearing. ${ }^{6}$ Endolymphatic sac surgery ? The controversy still rages. $^{78}$

We certainly agree on the need for cooperation between specialties in the management of patients with dizziness. Neurologists needs to work with otolaryngologists who have expertise and interest in otology. The neurologists of Leeds should know how lucky they are to have one.

G MICHAEL HALMAGYI PHILLIP D CREMER

Royal Prince Alfred Hospital, Sydney, Australia Correspondence to: Dr G Michael Halmagyi

1 Sass K Sensitivity and specificity of transtympanic electrocochleography in Menierè's disease. Acta Otolaryngol (Stockh) 1998;118:150-6.

2 Morrison GA, Sterkers JM. Unusual presentations of acoustic tumor. Clin Otolaryngol 1996; 21:80-3.

3 Halmagyi GM, Yavor RA, McGarvie LM. Tests of vestibular function. In: Jerger J, Alford B, Jenkins $\mathrm{H}$, eds. Electrophysiologic evaluation in otolaryngology. Basel, Karger, 1997;53:132-54. 